\title{
Robust Controller Design for a Nonlinear CSTR
}

\author{
Francis J. Doyle III, Andrew K. Packard; Manfred Morari ${ }^{\dagger}$ \\ Chemical Engineering 210-41 \\ California Institute of Technology \\ Pasadena CA 91125
}

\begin{abstract}
A design methodology is presented for the analysis and synthesis of robust linear controllers for a nonlinear continuous stirred tank reactor. Regions are defined in the phase plane in which the maintenance of robust stability and the achievement of robust performance levels are guaranteed. The results are based upon new extensions of the structured singular value theory to a class of nonlinear and time-varying systems.
\end{abstract}

\section{Introduction}

The inherently nonlinear nature of chemical processes has prompted considerable interest in nonlinear process control theory over the past decade. The complex dynamics which can evolve from these systems are revealed in the simple example of a single first order reaction carried out in a stirred tank. This system is known to exhibit bifurcations to multiple steady states and periodic limit cycles. Traditionally, the control schemes used for these nonlinear processes include "conservative" linear controllers and linear controllers with gain schedules. These methods are based on a first order approximation of the actual system at a single point and a discrete set of operating points, respectively. Consequently, these techniques cannot account for large perturbations or operation away from the steady state operating curve. In addition, linear model-based techniques have been used in the analysis of control schemes for such processes. Robustness properties have been calculated with respect to linear perturbations acting on a nominal linear model.

New advances in SSV theory allow the application of the results to a class of time-varying and nonlinear models. These models include the class of dynamical systems with conebounded nonlinearities. The focus of the present work is the application of this new technique to the calculation of margins of robust stability and robust performance for a nonlinear CSTR model. There have been only two published accounts of the application of these advances to practical control systems ([11],[3]); the latter paper contains the details of the work discussed here. Although the treatment will be developed for the generic class of systems with cone-bounded nonlinearities, it will be motivated by the specific application to a first order exothermic reaction carried out in a CSTR.

\footnotetext{
- Electrical Engineering

'Author to whom correspondence should be addressed: mm\%imcoromeo.caltech.eda
}

\section{The CSTR Problem Formulation}

\subsection{The Physical System}

The mass and energy balances for a CSTR with first order, irreversible, exothermic kinetics $(A \rightarrow B)$ are given by:

$$
\begin{gathered}
\dot{x}_{1}=-x_{1}+\mathcal{D a}\left(1-x_{1}\right) e^{\frac{x_{2}}{1+x_{2} / \gamma}} \\
\dot{x}_{2}=-x_{2}+\mathbf{B} \mathcal{D a}\left(1-x_{1}\right) e^{\frac{x_{2}}{1+x_{2} / \gamma}}-\beta\left(x_{2}-x_{c}\right)+\beta u+d
\end{gathered}
$$

(using the dimensionless quantities defined in the nomenclature). This simple model has two state variables (reactant concentration, reactor temperature) and one manipulated variable (cooling water temperature).

\subsection{Previous Nonlinear Approaches}

Recent advances in differential geometry have led to a number of useful, so called "linearization" techniques which have been applied to CSTR control. One approach [5] involves coordinate transformations on the state and input variables to transform the nonlinear system to a controllable linear system. Another approach [6] utilizes an input transformation by state feedback to obtain, in the new coordinates, a linear input/output map. However, the class of systems which yield solutions to state linearization is limited to involutive systems and input/output linearization requires minimum phase systems. Typical chemical reactor problems violate one or the other of these conditions.

A shortcoming of these techniques is the lack of robust performance guarantees. There are a few results, using numerical Lyapunov functions, which guarantee robust stability for certain unmodeled dynamics [6]. However, due to the "pseudo" transformed variables, it is not a straightforward procedure to specify even nominal performance criteria on the input, output and state variables for a given problem.

The results presented in this paper provide a complementary approach to the previous CSTR nonlinear control techniques by outlining a new technique for analyzing the robustness properties of nonlinear systems. The present work focuses on fairly simple linear controllers, but the theory is sufficiently general as to include a wide variety of nonlinear plants and nonlinear controllers.

\section{Structured Singular Value Concepts}

\subsection{The General Framework}

We will employ a general dynamical system description which establishes the relationship between the inputs, states and outputs. An uncertainty block is used to represent a family of plants which can be arrived at by perturbing the nominal 

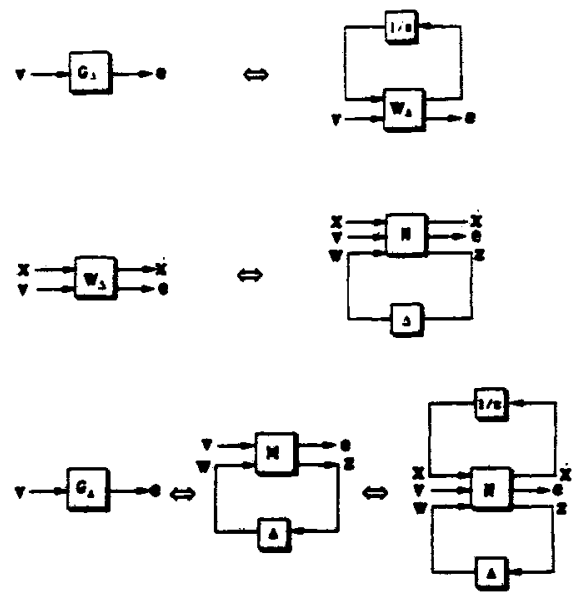

Figure 1: The General Framework (Perturbed)

plant. The input $v$ represents set points, disturbances and noise, the output $e$ represents error signals. In this framework, the control analysis problem focuses on two key questions: first, is the system stable for all perturbations in some prescribed set (robust stability); and second, does the error $e$ remain in a desired bounded set for all perturbations and inputs in some appropriate sets (robust performance).

The usual Euclidean norm or 2-norm will be used to calculate the norm of vectors in $\boldsymbol{C}^{n}$ or $\mathcal{R}^{n}$. For vector signals $e(t)$ this norm is defined to be: $\|e(t)\|^{2}=\int_{-\infty}^{\infty} e^{T}(t) e(t) d t$. The operator norm induced by the 2-norm is:

$$
\sup _{\substack{x \neq 0 \\ v \in L_{2}}} \frac{\|G v\|}{\|v\|}=\sup _{\omega} \bar{\sigma}(G(j \omega)) \triangleq\|G\|_{\infty}
$$

where $\mathcal{L}_{2}$ is the space of functions with bounded 2-norm.

An understanding of uncertainty descriptions can be gained by considering the nominal time-invariant, linear system $G(s)$ :

$$
\begin{aligned}
& \dot{x}=A x+B v \\
& e=C x+D v
\end{aligned}
$$

This system leads to a standard state-space realization:

$$
\left(\begin{array}{l}
\dot{x} \\
e
\end{array}\right)=\left(\begin{array}{ll}
A & B \\
C & D
\end{array}\right)\left(\begin{array}{l}
x \\
v
\end{array}\right)=W\left(\begin{array}{l}
x \\
v
\end{array}\right)
$$

It can be seen that $G(s)$ is obtained by closing a loop above $W$ with an integrator block $s^{-1}$, leading to the linear fractional transformation (LFT) for the transfer function:

$$
e=G(s) v=F_{u}\left(W, \frac{1}{s}\right) v=\left[D+C \frac{1}{s}\left(I-A \frac{1}{s}\right)^{-1} B\right] v
$$

where the subscript $u$ refers to the fact that the upper loop has been closed.

In a completely analogous fashion, it is possible to treat structured perturbations acting on a nominal system. Now we consider the perturbed state space realization, $W_{\Delta}$, and the subsequent LFT: $e=F_{u}\left(W_{\Delta}, \frac{1}{4}\right) v$. It is desirable to isolate the uncertainty elements from the overall transfer function $G_{\Delta}$. Stepwise, this proceeds as illustrated in Figure 1. First, matrix $W_{\Delta}$ is rewritten as a feedback connection of a matrix $N$ and an uncertainty block $\Delta$. The structure of the uncertainty block is a key feature of this representation and will be exploited in the following sections. In general, the uncertainty block will be a linear operator in the set:

$$
\Delta:=\left\{\operatorname{diag}\left[\delta_{1} I_{r_{1}}, \ldots, \delta_{m} I_{r_{m}}, \Delta_{1}, \ldots, \Delta_{n}\right]\right\}
$$

where depending on the problem the $\delta_{i}, \Delta_{i}$ will be restricted to certain classes. We define the bounded sabset:

$$
B \Delta:=\{\Delta \in \Delta \mid \sigma(\Delta) \leq 1\}
$$

Finally, a general LFT for $G_{\Delta}(s)$ can be given by: $G_{\Delta}(s)=$ $F_{\ell}\left(F_{w}\left(N, \frac{1}{6}\right), \Delta\right)$. Defining $M \triangleq F_{v}\left(N, \frac{1}{6}\right)$ (see Figure 1) yields: $G_{\Delta}(s)=F_{t}(M, \Delta)$. Thus, the so-called " $M-\Delta$ structure" is constructed. This mapping can be appropriately partitioned:

$$
\left(\begin{array}{l}
e \\
z
\end{array}\right)=\left(\begin{array}{ll}
M_{11} & M_{12} \\
M_{21} & M_{22}
\end{array}\right)\left(\begin{array}{c}
v \\
w
\end{array}\right)
$$

For $w=\Delta x$, the general LFT for $G_{\Delta}$ is given by:

$$
F_{C}(M, \Delta) \triangleq M_{11}+M_{12} \Delta\left(I-M_{22} \Delta\right)^{-1} M_{21}
$$

In this LFT framework, robust stability requires that $F_{l}(M, \Delta)$ remain stable for all $\Delta \in B \Delta$. Robust performance requires that a norm bound on the mapping from input $v$ to output $e$ is met for all perturbations.

Filters can be designed which "shape" the input signal class into the expected set of inputs, and similarly weight the outputs (by frequency) according to the specified performance criteria. All of these "performance and uncertainty weights" are usually absorbed into the structure $M$. Then an appropriate robust performance condition is:

$$
\left\|F_{\ell}(M, \Delta)\right\|_{\infty}<1 \quad \forall \Delta \in B \Delta
$$

\subsection{Benefit of Constant D Scalings}

Considering the system in Eq. 10, it is clear that the inpetoutput system is stable if the operator $\left(I-M_{22} \Delta\right)^{-1}$ is stable. This stability result can be reformulated by applying the small gain theorem. This gives a sufficient condition for stability:

$$
\sup _{\omega} \bar{\sigma}\left(M_{22}\right)<\gamma \quad(\gamma>0) \quad \forall \Delta \in \frac{1}{\gamma} B \Delta
$$

where $\frac{1}{7} B \Delta$ is the subset of $\Delta$ with $\bar{\sigma}(\Delta) \leq \frac{1}{\gamma}$.

Consider the class of matrices, $D$, which commute with the perturbation block $\Delta$. If $D$ and $\Delta$ commute, then by definition $D \Delta D^{-1}=\Delta$. If this commutivity holds, then for any operator $M_{22}$, the two loops in Figure 2 are equivalent to each other. Now, application of the small gain theorem to Figure 2 guarantees the stability of the loop for all stable and time-varying $\Delta$ which also satisfy:

$$
\bar{\sigma}(\Delta)<\frac{1}{\left\|D M_{22} D^{-1}\right\|_{\infty}}
$$

This equation shows that conservatism can be minimized by considering:

$$
\inf _{D \in \mathcal{D}}\left\|D M_{22} D^{-1}\right\|_{\infty}
$$

where $\mathcal{D}$ defines an appropriate commuting set for the $\Delta$ in Eq. 7:

$$
\begin{aligned}
\mathcal{D} \triangleq & \left\{\operatorname{diag}\left[D_{1}, \ldots, D_{m}, d_{1} I_{k_{1}}, \ldots, d_{n} I_{k_{n}}\right]\right. \\
& \mid D_{i} \in C^{T_{i} \times r_{i}} \text { is invertible, } d_{i} \neq 0
\end{aligned}
$$

For instance, time-varying complex $\Delta$ commute with constant $D$. The $D$ scale which achieves or gets arbitrarily close to the infimum in Eq. 14 is termed the optimal $D$ scale since it maximizes the bound that can be obtained with this method. 


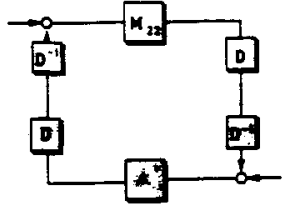

8

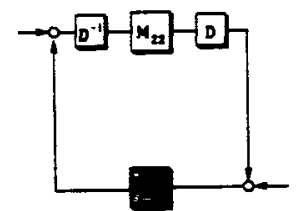

Figure 2: Equivalent Scaled Loops for Robust Stability

Given these $D$ scales, it is possible to formulate a less conservative condition for robust stability than that propased in the previous subsection:

Theorem 1(Stability; Time-Varying, Complex $\Delta$ )

The system $F_{\ell}(M, \Delta)$ is stable for all $\Delta \in B \Delta$ if

$$
\inf _{D} \sup _{\omega} \bar{\sigma}\left(D M_{22} D^{-1}\right)<1
$$

where $D$ is the appropriate commuting matrix for $\Delta$.

Just as for robust stability, a less conservative condition than Eq. 11 can be derived for robust performance by absorbing the $D$ scales into the $M$ block:

Theorem 2(Performance; Time-Varying, Complex $\Delta$ ) $F_{\ell}(M, \Delta)$ is stable and $\left\|F_{\ell}(M, \Delta)\right\|_{\infty}<1 \quad \forall \Delta \in B \Delta$ if

$$
\underset{D}{\inf \sup _{\omega} \tilde{\sigma}}\left[\left(\begin{array}{ll}
I & 0 \\
0 & D
\end{array}\right) M\left(\begin{array}{cc}
I & 0 \\
0 & D^{-1}
\end{array}\right)\right]=\beta<1
$$

where $D$ is the appropriate commuting matrix for $\Delta$.

In order to use Thms. 1 and 2 a procedure has to be found for finding the optimal $D$ 's in Eqs. 16 and 17. The following two sections establish an equivalent minimization problem which is easier to solve.

\subsection{Connection to Lyapunov Approach}

This section outlines the robust stability and robust performance results in the time domain. The motivation for this analysis is twofold: first, the usual Lyapunov results for stability can be clearly represented in the time domain; and second, the actual calculations involved for the scaled singular values are computationally more attractive in this domain.

Consider the linear difference equation:

$$
x_{k+1}=F_{\ell}\left(\tilde{N}, \Delta_{k}\right) x_{k}
$$

where $x_{k} \in \mathcal{C}^{n}, \tilde{N} \in \mathcal{C}^{(n+m) \times(n+m)}$ and for each $k, \Delta_{k}$ is an element of the prescribed uncertainty set $\Delta . \Delta_{k}$ is assumed to vary with $k$ and $\bar{\sigma}\left(\Delta_{k}\right) \leq 1$. A necessary and sufficient condition for the existence of a single quadratic Lyapunov function, $x^{*} P x$, for the entire set of operators contained in $\left\{F_{\ell}\left(\tilde{N}, \Delta_{k}\right) \mid \Delta_{k} \in B \Delta\right\}$ is given by:

$$
\max _{\Delta_{k} \in B \Delta} \bar{\sigma}\left(T F_{\ell}\left(\tilde{N}, \Delta_{k}\right) T^{-1}\right)<1
$$

for some $T \in \mathcal{C}^{n \times n}$, invertible
This result is equivalent to the usual discrete Lyapunov matrix equation, where $P=T^{*} T$ is the resultant Lyapunov function. Comparing (16) and (19) it is clear that the Lyapunov approach involves a type of scaling not unlike the optimal $D$ scales. In this case, the "scaling" consists of a coordinate transformation $T$ on the state variable. The conservatism of the result in Equation 19 can be reduced by introducing the appropriate D-scalings. Now, a sufficient condition for the existence of a Lyapunov function is given by:

$$
\inf _{T, D_{2}} \bar{\sigma}\left[\left(\begin{array}{cc}
T & 0 \\
0 & D_{2}
\end{array}\right)\left(\begin{array}{cc}
\tilde{N}_{11} & \tilde{N}_{12} \\
\tilde{N}_{21} & \tilde{N}_{22}
\end{array}\right)\left(\begin{array}{cc}
T^{-1} & 0 \\
0 & D_{2}^{-1}
\end{array}\right)\right]=\beta<1
$$

where $D_{2}$ commutes with $\Delta_{k}$ and $T \in C^{n \times n}$, invertible. For a limited, special class of uncertainties, Eq. 20 is also necessary for the existence of a single quadratic Lyapunov function [9]. For readers familiar with structured singular value theory, this class is precisely those problems for which the structured singular value is equal to its upper bound [10]. It can be verified that the uncertainty description used in the subsequent reactor analysis is a member of this set and thus the stability results for the reactor problem are completely equivalent to quadratic Lyapunov function stability results.

The robust performance results follow quite naturally from the above calculations. Consider the full system:

$$
\left(\begin{array}{c}
x_{k+1} \\
e_{k} \\
z_{k}
\end{array}\right)=\left(\begin{array}{lll}
N_{11} & N_{12} & N_{13} \\
N_{21} & N_{22} & N_{23} \\
N_{31} & N_{32} & N_{33}
\end{array}\right)\left(\begin{array}{c}
x_{k} \\
v_{k} \\
w_{k}
\end{array}\right)
$$

where the uncertainty is fed back from $z_{k}$ to $w_{k}$ through the perturbation block $\Delta_{k}$. The objective is the achievement of gain less than 1 across the $v$ to $e$ path for all perturbations in the set $\Delta_{k}$. Robust performance can be guaranteed if for all $\Delta_{k} \in B \Delta$ if

$$
\inf _{T, D} \bar{\sigma}\left[\left(\begin{array}{ccc}
T & 0 & 0 \\
0 & I & 0 \\
0 & 0 & D
\end{array}\right) N\left(\begin{array}{ccc}
T^{-1} & 0 & 0 \\
0 & I & 0 \\
0 & 0 & D^{-1}
\end{array}\right)\right]=\beta<1
$$

Defining $\ell_{2}$ as the set of 2 -norm bounded discrete signal:. then equation 22 implies the robust performance result:

$$
\text { if Eq. } 22 \text { and } x_{0}=0 \text { and }\left\{v_{k}\right\}_{k=0}^{\infty} \in \ell_{2} \text { then }\|e\| \leq \beta\|v\|
$$

The result given in equation 22 can also be interpreted in terms of the so-called power norm. For vector signals $e(t)$ the power norm is defined by: $\|e(t)\|_{P}^{2}=$ $\lim _{\tau \rightarrow \infty} \int_{-\tau}^{\tau} \frac{1}{2 \tau} e^{T}(t) e(t) d t$. Strictly speaking the power norm does not satisfy all the properties of a norm, however the class of bounded power signals is of practical interest. Defining $\ell_{P}$ as the set of power norm bounded discrete signals, then the appropriate robust performance result is:

$$
\text { if Eq. } 22 \text { and }\left\{v_{k}\right\}_{k=0}^{\infty} \in \ell_{P} \text { then }\|e\|_{P} \leq \beta\|v\|_{P}
$$

\subsection{Computation of Optimal Constant $D s$}

The unifying connection between the continuous frequency domain results of Section 3.2 and the discrete time domain results of Section 3.3 can be given by a result which states that constant $D$ scalings can be found if and only if the state space test can be made. The latter minimization can be reformulated as a convex, nondifferentiable optimization, and as such has been computationally successful [1]. For a complete proof of this result, the reader is referred to [10].

A final remark should be made concerning the results of the previous sections. For the special case of linear time-invariant 
perturbations, there exist necessary and sufficient conditions for robust stability and robust performance. The results involve a test on the so-called structured singular value, for which the $D$ scaled maximum singular value is an upper bound. The reader is referred to [4] for further details. In addition, theoretical results have been obtained for real perturbations [2]. This method shares some of the computationally attractive features of the previously discussed problems.

\subsection{Conic-Sector Bounded Nonlinearities}

In this section, a class of nonlinear systems is described which fits into the previously described $M-\Delta$ structure. A conic sector is defined to be:

$$
\operatorname{Cone}(C, R) \equiv\{(x, y)\|\| y-C x\|\leq\| R x \|\}
$$

where $(x, y)$ is the input, output pair for the operator. Evidently, a nonlinear operator enveloped tightly by a conic sector is most accurately approximated (linearly) by the cone center $C$. In general, the cone center will not coincide with the plant described by the Jacobian of the nonlinear model evaluated at an operating point.

Note that because we have replaced a potentially highly nonlinear operator by a function consisting of two linear timeinvariant operators, the cost of this simplification is increased conservatism. The Cone $(C, R)$ describes many input-output pairs, some of which may yield poorer performance than the original operator.

It should be clear that there exists a direct correspondence between a nonlinear cone-bounded operator and a time-varying gain. From the conic sector definition, the plant can be interpreted as being equal to the nominal value $(C)$ which is perturbed by a time varying gain of magnitude $R$. $R$ and $C$ are absorbed into the system to arrive at the general uncertainty structure in Figure. 1 where now $\Delta$ is a time-varying gain of norm one.

The details of the constraction of interconnection structures for general uncertainty descriptions can be found in [8].

\section{Controller Design for a Nonlinear CSTR Model}

The case study under consideration is regulation of outlet reactant concentration, which is assumed to be measured. Coolant temperature is the manipulated variable; input saturations will not be considered. The control objective will be the accurate tracking of step changes in the concentration set point. Two sets of parameter values will be investigated, the first being ( $B=22 ., \beta=3.0, \gamma \rightarrow \infty, \mathcal{D}_{a}=0.082$ ). These values yield an open-loop system with 3 steady states (1 stable, 2 unstable) and an attracting limit cycle. The other $\left(B=1.0, \beta=0.3, \gamma=20.0, D_{a}=0.072\right)$ yields an open-loop system with a single stable steady state.

\subsection{Uncertainty Characterization}

First, the CSTR dynamics are represented in deviation variables so that the origin is a fixed point. The equations are:

$$
\begin{aligned}
\dot{\tilde{x}}_{1}= & \tilde{f}_{1}=-\bar{x}_{1}-x_{10}+\mathcal{D} a\left(1-\tilde{x}_{1}-x_{10}\right) e^{\frac{z_{2}+x_{20}}{1+\left(\dot{z}_{2}+x_{20}\right) / \gamma}} \\
\dot{\bar{x}}_{2}= & \tilde{f}_{2}-\beta u=-\tilde{x}_{2}-x_{20}-\beta\left(\tilde{u}+u_{0}\right) \\
& +\mathrm{BD} a\left(1-\tilde{x}_{1}-x_{10}\right) e^{\frac{\dot{z}_{2}+x_{20}}{1+\left(x_{2}+x_{20}\right) / \gamma}}-\beta\left(\tilde{x}_{2}+x_{20}\right)
\end{aligned}
$$

The nonlinearity in these equations is solely a function of the state variables and therefore would be equivalent to uncertainty in the state-space $A$ matrix, provided that $\tilde{f}_{1}, \tilde{f}_{2}$ can be shown to be conic-sector bounded.

For the purposes of this study we will impose bounds on the state variables which define an operating window in the phase-plane. The region will consist of a scaled unit ball which is equivalent to an ellipse in the original phase space. It can be verified that a bounding of the state variables is possible by considering the result implied by Eq. 22 :

$$
\|e\|^{2}+\left(1-\beta^{2}\right)\|x\|^{2} \leq \beta^{2}\|v\|^{2}+\left\|x_{0}\right\|^{2}
$$

In fact, a less conservative result is obtained using a subsystem of the original $N$ matrix consisting of the first and last rows (remove the $e$ term from the calculations). This yields a relationship between the bound on the states and both the initial conditions and input bound:

$$
\|x\|^{2} \leq \frac{\beta^{2}\|v\|^{2}+\left\|x_{0}\right\|^{2}}{1-\beta^{2}}
$$

It is easy to show that for a state bound of radius 1 , one can define a tolerable bound on $x_{0}$ for zero input equal to $\sqrt{1-\beta^{2}}$, and a bound on $x_{0}$ for unit bounded input equal to $\sqrt{1-2 \beta^{2}}$. These expressions suggest an iterative algorithm for the selection of the state bound. The size of the ball for $\|x\|$ is shronk until a scaled singular value $(\beta)$ is obtained which is less than $\sqrt{.5}$. It is possible to shrink this bound even further to alter the relative sizes of the two bounds on $x_{0}$.

A careful analysis of the problem reveals that it is possible to formulate a tight conic sector bound on $\tilde{f}_{1}$ and $\tilde{f}_{2}$ with only one uncertain gain. This is due to the linear dependence of the rate expression on the concentration. This can be seen in a three-dimensional plot, (Figure 3), where with the proper perspective on the $x_{1}-x_{2}$ plane, the nonlinearity falls within a well-defined "bow-tie" region. The nonlinearity is bounded by two planes, both perpendicular to the plane of observation in the figure. The final plant description is given by:

$$
G_{\Delta}:\left(\begin{array}{l}
\dot{x} \\
y
\end{array}\right)=\left[\left(\begin{array}{cc}
\bar{A} & B \\
C & 0
\end{array}\right)+\Delta_{1}\left(\begin{array}{cc}
\tilde{A}_{1} & 0 \\
0 & 0
\end{array}\right)\right]\left(\begin{array}{l}
x \\
u
\end{array}\right)
$$

where $\tilde{A}, \tilde{A}_{1}$ are easily calculated by projecting the elliptic state bounds in the $x_{1}-x_{2}$ plane onto the nonlinearities $\tilde{f}_{1}$ and $\tilde{f}_{2}$.

\section{2 $\mathrm{H}_{2}$-Optimal Controller Design}

A convenient choice of control objective is the minimization of the integral square error (ISE) or the 2-norm of the error for a specific input (e.g., a step input). Following the IMC procedure [8], one can readily derive the $H_{2}$-optimal controller. The procedure requires a nominal linear plant, which will be taken to be the center of the cone defined by the chosen operating window. If this model is designated as $P_{m}(s)$, then the controller is given by $P_{m}^{-1}(s) F(s)$. Here $F(s)$ is a low-pass filter which renders the controller proper, allows for asymptotic tracking properties, and provides robustness.

\section{3 $H_{\infty}$-Optimal Controller Design}

For the unstable operating condition, we will consider, as an alternative to the $\mathrm{H}_{2}$-optimal control objective, the minimization of the error for a set of inputs of bounded 2-norm. 


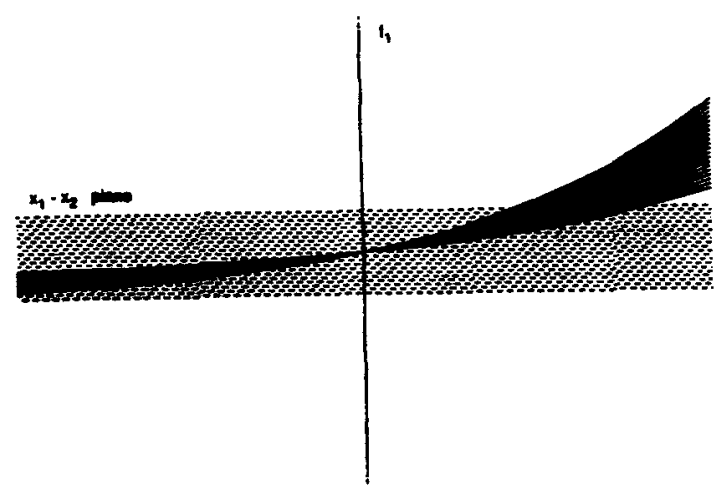

Figure 3: Three Dimensional Perspective of Nonlinearity

Consequently this optimization entails a minimization of the infinity-norm of the weighted sensitivity operator. An optimal method can be used to find the controller, $K$, which solves:

$$
\left\|F_{\ell}(M, \Delta)\right\|_{\infty} \leq 1
$$

where $M$ represents the weighted closed loop sensitivity function. The optimal $K$ is found by a search over all stabilizing $K$ 's and scaling $D$ 's so that the quantity $\left\|D F_{\ell}(M, \Delta) D^{-1}\right\|_{\infty}$ is minimized.

\section{Computational Results}

\subsection{Single, Stable Steady State}

For this case study, a relatively mild operating condition was considered with the expectation that robustness properties can be established for a large window in the phase plane. The operating window is defined to be: $\left(\frac{x_{1}-0.3}{0.3}\right)^{2}+\left(\frac{x_{2}-1.06}{1.96}\right)^{2}=1$. An analysis of the robust stability of the closed loop system yields a scaled maximum singular value of 0.69 for the IMC controller. Thus stability is assured throughout the entire operating window provided that appropriate bounds are defined on the initial conditions (next section). An analytical performance eviluation of the controller is shown in the performance degradation plots of Figure 4. The intercept of the curve at the $y$-axis represents the nominal performance (zero perturbation) while perturbation magnitude corresponding to the vertical asymptote represents the inverse of the SSV for robust stability (no performance). Another interpretation of the $x$-axis is the necessary reduction factor for the window to achieve a given performance level. An additional curve is included in this plot to represent the necessary and sufficient condition for robust performance with time-invariant perturbations. The distance between the two curves indicates both the sufficiency of the time-varying result and the additional constraint that time-varying perturbations place on performance levels above those imposed by time-invariant perturbations. For this case, there is negligible difference.

\subsection{Multiple Steady States}

The second case study presents a more formidable control challenge. Consequently, both $H_{2}$ and $H_{\infty}$-optimal controllers have been designed for this case study. For the

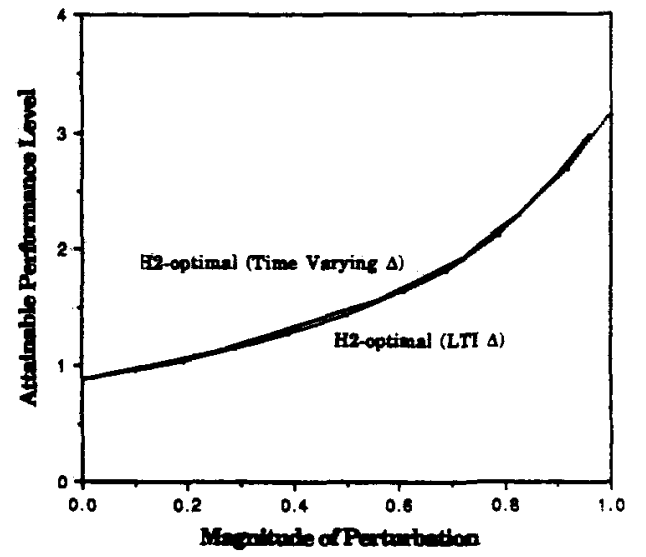

Figure 4: Performance Degradation Curve (Stable Case)

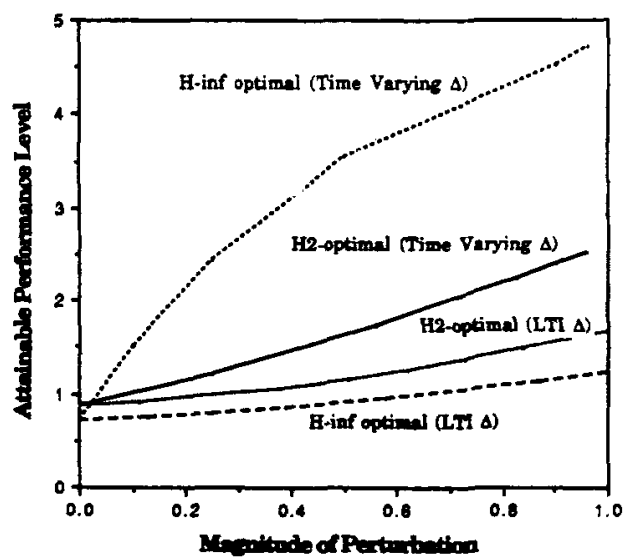

Figure 5: Performance Degradation Curve (Unstable Case)

chosen parameter values, the open-loop dynamics are somewhat pathological. In this case, the control objective is the construction of a window about the unstable open loop operating point $\left(x_{1}=0.305, x_{2}=1.677, u=0\right)$. The chosen operating region is: $\left(\frac{x_{1}-0.305}{0.15}\right)^{2}+\left(\frac{x_{2}-1.677}{1.0}\right)^{2}=1$. This window also encompasses the stable uncontrolled fixed point $x_{1}=0.184, x_{2}=1.014$.

Robust stability analysis yields scaled maximum singular values of 0.49 for the IMC controller and .60 for the $H_{\infty}$ optimal controller. In this case, both the $H_{2}$-optimal and the $H_{\infty}$-optimal closed loops have guaranteed stability throughout the phase window.

The performance degradation curves for these two controllers are given in Figure 5. The distance between the timevarying and linear-time-invariant curves are more significant for this pathological set of parameters.

\subsection{Interpretation of Results}

In order to accurately interpret the robustness results of the previous section, it is useful to recall the relationship defined in Eq. 26. Depicted in Figures 6 and 7 are the appropriate bounds for the stable and unstable operating point, respectively. For the stable operating point, the requisite bound on the initial conditions for zero input is: $\left(\frac{x_{1}-0.3}{0.22}\right)^{2}+\left(\frac{x_{2}-1.96}{1.23}\right)^{2}=1$; and for unit bounded input: $\left(\frac{x_{1}=0.3}{0.066}\right)^{2}+\left(\frac{z_{2}-1: 3}{0.43}\right)^{2}=1$. Similarly, one obtains the following 


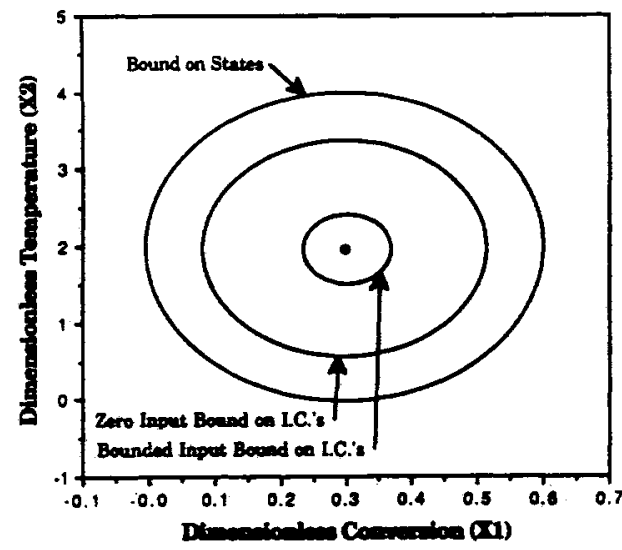

Figure 6: Stability Regions (Stable Case)

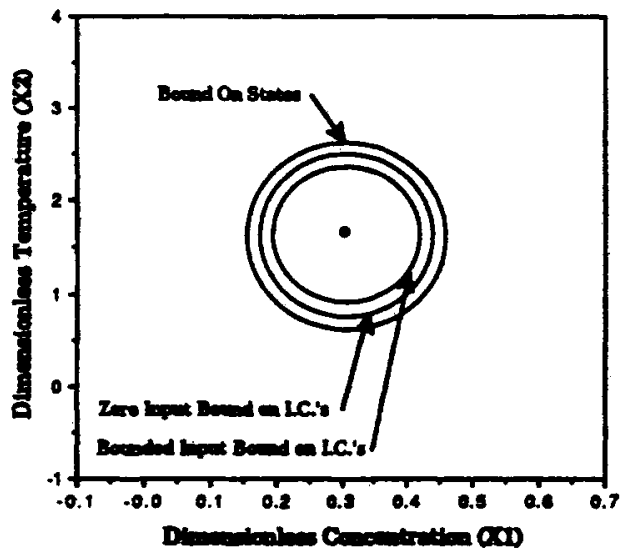

Figure 7: Stability Regions (Unatable Case)

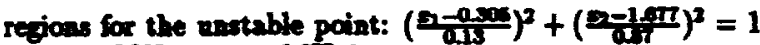

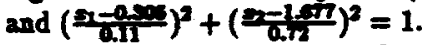

Using standard Lyapunor-function techriques, it is possible to construct rejions of attraction for the cloced loop CSTR. Not unexpectedly, theoe regions are considerably larger than the ellipees for the cones in Figures 6 and 7. The large disparity can be readily explained by the fact that the cone contains many nonlinearities, some of which are evidently more pathological than the original system. The ntility of this method is a computationally attractive scheme for calculating regions for bounded energy and bounded power performance results. As opposed to linear methods which yield performance results in an ambiguous region "in which the linear model is valid", our technique yields an explicit lower bound on the appropriate regions.

\section{Conclusions}

A practical problem is presented which demonstrates the utility of the new extensions of the structured singular value to nonlinear systems. While robust control of the closedloop system over the entire phase plane is too ambitious for these potentially pathological systems, it is demonstrated that closed loop systems can be obtained with linear controllers which are robust over a large portion of the phase plane.
While conservatism is inherent in the analysis of timevarying systems, the presented technique shows a computationally attractive method for quadratic Lyapunov function construction. The conservatism of these results could be further reduced by considering real variations in $\Delta$ (as opposed to complex).

\section{References}

[1] Boyd, S. and Desoer, C. A., "Subharmonic Functions and Performance Bounds on Linear Time Invariant Feedback Systems”, UC Berkeley/ERL Memo M84/51, 1984.

[2] Boyd, S. and Yang, Q., "Structured and Simultaneous Lyapunor Functions for System Stability Problems", Stanford University/Information Systems Laboratory Technical Report No. L-104-88-1, 1988.

[3] Doyle III, F. J., A. P. Pachord and M. Morari, "Robust Controller Dexige for a Nonlinear CSTR", Chem. Eng. Sci., in press, 1989.

[4] Doyle, J. C., "Analysis of Feedback Systems with Structured Uncertainties" IEEE Proc., Part D, 6, 242-250, 1982.

[5] Hoo, K. A. and Kantor, J.C., "An Exothermic Continnous Stirred Tank Reactor is Feedback Equivalent to a Linear System", Chem. Eng. Comm., 37, 1-10, 1985.

[6] Kravaris, C. and Palanki, S., "Robuot Nonlinear State Feedback Under Structured Uncertainty", AIChE J., 7, 1119-1127, 1988.

[7] Kravaris, C. and Kantor, J.C., "Geometric Methods for Nonlinear Process Control: A Tutorial", I. \& E.C. Res., in preparation, 1989.

[8] Morari, M. and Zafirion, E., Robuet Process Controh, Prentice-Hell Inc., Englewood Ciffe, NJ, 1989.

[9] Pactard, A., "What's New with $\mu$ : Strectured Uncertainty in Multivariable Coatrol", Ph.D. Thexis, Univer. sity of California, Berkeley, 1987.

[10] Pachard, A. and Doyle, J., "Structured Singular Valve with Repeated Scalar Blocks", Proc. 1988 Am. Coatr. Conf., 1213-1218.

[11] Smith, R.S. and Doyle, J., "The Two Tank Experiment: A Benchmark Control Problem”, Proc. 1988 Am. Contr. Conf., 2026-2031.

\section{Nomenclature}

$\begin{array}{llll}B & -\Delta H C_{A s} / C_{p} T_{f_{0}} \gamma & T_{\infty} & \text { nominal coolant temp } \\ C_{A} & \text { reactant conc. } & T_{f} & \text { actual feed temp } \\ C_{p} & \text { heat capacity } & T_{f_{0}} & \text { nominal feed temp } \\ \mathcal{D} a_{a} & \frac{y}{Q_{1}} K_{0} \mathrm{e}^{-\gamma} & U & \text { heat transfer coeff. } \\ d & \left(T_{f}-T_{f_{0}}\right) / T_{f_{0} \gamma} & V & \text { reactor volume } \\ E a & \text { activation energy } & u & \left(T_{c}-T_{\infty_{0}}\right) / T_{f_{0} \gamma} \\ \Delta H & \text { heat of reaction } & x_{1} & \left(C_{A f}-C_{A}\right) / C_{A} \\ k_{0} & \text { rate constant } & x_{3} & \left(T-T_{f_{0}}\right) / T_{f_{0}} \gamma \\ Q_{f} & \text { feed stream flow rate } & & \\ R & \text { ideal gas constant } & \beta & U A_{h} / Q_{f} C_{p} \\ T_{c} & \text { coolant temp } & \gamma & E a / R T_{f_{0}}\end{array}$

